\title{
Cognitive function in schizophrenia: insights from intelligence research*
}

Eileen M. Joyce

\section{Summary}

schizophrenia is characterised by generalised cognitive impairment that is both a risk factor and a predictor of outcome. Recent research into human intelligence supports the view that, in schizophrenia, poor performance on disparate cognitive tasks can be explained by dysfunction of a frontoparietal neural network thought to support fluid intelligence.

\section{Declaration of interest}

None.
Eileen M. Joyce is Professor of Neuropsychiatry at the UCL Institute of Neurology. Her research concerns cognitive impairments in first-episode schizophrenia in relation to clinical outcomes and neurobiological abnormalities.

Nearly 20 years of research has failed to elucidate a defining 'cognitive signature' of schizophrenia. Instead, the most replicated finding is of impairment in virtually every cognitive domain that differs between individuals in degree but not specificity. ${ }^{1}$ The now widely held view, that cognitive impairment in schizophrenia is generalised, and the observation that it is present at psychosis onset $^{2}$ and is more strongly correlated with functional outcomes than symptoms, ${ }^{3}$ suggests that cognitive function should be introduced into clinical practice as an important dimension of the disorder. The growing recognition that there are psychological interventions that improve general cognition in schizophrenia ${ }^{4}$ makes this a particularly timely and important consideration.

However, a more detailed account of the general performance decrement in schizophrenia is still warranted. This is because it will have implications for our understanding of the disorder as a whole. Replicated studies have shown that people who develop schizophrenia tend to perform worse than their healthy peers on scholastic or IQ tests before psychosis onset and as far back as childhood. Importantly, the severity of this decrement is linearly related to the likelihood of developing psychosis, suggesting that the process mediating cognitive impairment is part of the general neurodevelopmental risk for schizophrenia. ${ }^{5}$ The aim of this article is to determine whether the generalised cognitive impairment in schizophrenia can be characterised further, particularly with respect to a neurobiological substrate, by reference to recent advances in intelligence research.

\section{The nature of the general cognitive impairment in schizophrenia}

Psychometricians have long recognised that the performance of healthy populations on batteries of diverse neuropsychological tests varies at three hierarchical levels. These are: ability on specific tests; ability on groups of tests assessing similar cognitive constructs (such as working memory, perceptual organisation); and general ability. ${ }^{6}$ General ability or $g$ is thought to contribute to the performance of all cognitive tests and approximately $50 \%$ of the variance in performance across tests can be accounted for by

*This editorial is based in part on The Paykel Lecture, University of Cambridge delivered in October 2012 differences at this level. ${ }^{6}$ Statistical accounts of neuropsychological performance further suggest that there are two highly intercorrelated components of $g$ : 'crystalline intelligence', derived from knowledge-based tests that rely on education and past experience, and 'fluid intelligence', calculated from tests of reasoning and rapid information processing. ${ }^{6}$ The general ability of individuals is commonly measured by instruments such as the Wechsler Adult Intelligence Scale (WAIS) ${ }^{7}$ that assess a wide range of abilities from which a composite score (IQ) is derived as well as the crystalline and fluid components denoted as verbal IQ and performance IQ respectively.

Recent research has investigated whether there is more to the concept of general ability than a statistical construct. In other words, whether $g$ is an entity characterised by a set of fundamental cognitive processes with a neural basis. In this respect, two things are noteworthy about neuropsychological test performance in schizophrenia. First, when compared with healthy populations, the difference in performance is mostly at the level of general ability. ${ }^{8}$ Second, performance is less differentiated than normal in that more of the within-group variation is accounted for by general than specific ability. ${ }^{8}$ This pattern of effects suggests that people with schizophrenia have a more limited general cognitive resource than average and that this constrains the performance of a wide range of specific cognitive functions. Arguably, if a specific neurobiological counterpart of this general resource can be identified, an important clue to the aetiology of schizophrenia will be revealed.

One lead concerns information-processing speed, which has been shown to explain up to $25 \%$ of the variance in the general ability of healthy populations. ${ }^{6}$ This is relevant because a metaanalysis of the performance of schizophrenia and control groups on a large number of different neuropsychological tests found that the greatest effect size for group difference was not for IQ but for a test of processing speed. ${ }^{9}$ In support of the importance of processing speed, several other studies have found that even when patients with schizophrenia and healthy controls are precisely matched for WAIS IQ, there is a mismatch in performance across WAIS subtests so that in schizophrenia, the processing speed domain is disproportionately impaired. ${ }^{10}$ This suggests that reduced processing speed may be one of the fundamental causes of the general cognitive impairment in schizophrenia. Regarding neurobiology, speed of processing is highly dependent on the integrity of myelinated axons. This can be measured in the brain with magnetic resonance diffusion tensor imaging. Using this methodology, a recent study of healthy people found that WAIS IQ correlated with an index of white matter integrity across 12 major white matter tracts. ${ }^{11}$ 
Importantly, structural equation modelling showed that this effect was fully mediated by information-processing speed measured separately with non-WAIS tasks. As there is now compelling evidence that white matter development is abnormal in schizophrenia, these findings provide a testable hypothesis for linking generalised cognitive impairment in schizophrenia to a neurodevelopment risk factor.

Other research into the neurobiological underpinnings of $g$ concerns executive function. This consists of a set of cognitive processes that serve to control behaviour in the face of changing environmental contingencies and includes working memory, attention switching and response inhibition. The cognitive requirements of tests of fluid intelligence overlap considerably with those of executive function and functional imaging studies have linked both fluid intelligence and executive function with a common neural substrate. Thus, performance accuracy on a working memory task at a point requiring maximal executive control was shown to depend on fluid intelligence ability on the one hand and the degree of engagement of specific areas in frontal and parietal cortex on the other. ${ }^{12}$ Human lesion studies have also determined that fluid intelligence is supported by brain regions corresponding to those identified in functional imaging studies, namely posterolateral frontal, dorsomedial frontal and midparietal cortex. In one important study the degree of volume loss within, but not outside, these regions was critically related to the loss of fluid intelligence. ${ }^{12}$ The implication of these and other studies is that there is a frontoparietal neural network that provides the processing requirements for the efficient execution of disparate cognitive tasks that enable the environmentally adaptive behaviour encapsulated in the concept of fluid intelligence. ${ }^{12-14}$

This hypothesis provides a unitary framework for reconciling the diverse cognitive findings in schizophrenia. That is, the dysfunction of a specific set of cortical areas and their white matter connections may account for a substantial amount of the cognitive impairment seen in this disorder. Illustrating this point is the finding of a meta-analysis of functional imaging studies that examined frontal lobe function in patients with schizophrenia and healthy controls. The studies that were evaluated used a variety of different cognitive tests but a common set of frontal cortical areas was activated each time. These conformed to nodes of the same neural network implicated in the support of intelligence. Importantly, although the same areas were activated in both groups, this was atypical in patients suggesting dysfunctional processing within this network. ${ }^{15}$

The brain abnormalities of schizophrenia admittedly extend well beyond this frontoparietal network and involve other cortical areas, subcortical structures and their white matter connections. Dysfunction of these different neural networks is also likely to account for other important facets of the disorder, such as impaired emotional and social cognition, and they ultimately may prove equally or more deleterious to everyday function than intellectual impairment. However, in terms of elucidating the neurobiological substrate of the disorder, the generalised cognitive impairment may be more tractable than these other problematic areas. This is because the cognitive processes mediated by the frontoparietal network lend themselves more to comparative and computational neuroscience. For example, Duncan has shown that the frontoparietal network is activated by diverse cognitive task requirements in healthy people. On the basis of this, he proposes that it serves as a 'multiple demand system' important for the 'programming of goal directed behaviour' subserving fluid intelligence. ${ }^{16}$ Using analogies with artificial intelligence and data from studies of non-human primate single-cell recordings and human functional neuroimaging, he provides evidence for how this system can exert cognitive control. This includes the fractionation of encountered tasks into essential subroutines, the switching of attention from one to another in a sequential manner and the extraction of important information from each step to pass onto to the next for successful completion of the programme.

\section{Implications}

An understanding of the core functions of this frontoparietal network, its neurobiology and how it is influenced during development may lead to important new insights into the causative abnormal mechanisms of schizophrenia. Such research may also elucidate whether the plasticity of this system is modifiable during adult life, for example by pharmacological or psychological means. If so, this will illuminate the search for much needed new methods of remediation.

Eileen M. Joyce, MA, PhD, MRCP, FRCPsych, Box 19, The National Hospit for Neurology and Neurosurgery, Queen Square, London WC1N 3BG, UK. Email: e.joyce@ucl.ac.uk

First received 7 Aug 2012, final revision 4 Apr 2013, accepted 15 Apr 2013

\section{Funding}

E.M.J. is supported by the UCLH/UCL Biomedical Research Centre.

\section{References}

1 Saykin AJ, Shtasel DL, Gur RE, Kester DB, Mozley LH, Stafiniak P, et al. Neuropsychological deficits in neuroleptic naive patients with first-episode schizophrenia. Arch Gen Psychiatry 1994; 51: 124-31.

2 Leeson VC, Sharma P, Harrison M, Ron MA, Barnes TR, Joyce EM. IQ Trajectory, cognitive reserve, and clinical outcome following a first episode of psychosis: a 3-year longitudinal study. Schizophr Bull 2011; 37: 768-77.

3 Green MF, Kern RS, Heaton RK. Longitudinal studies of cognition and functional outcome in schizophrenia: implications for MATRICS. Schizophr Res 2004; 72: 41-51.

4 Wykes T, Huddy V, Cellard C, McGurk SR, Czobor P. A meta-analysis of cognitive remediation for schizophrenia: methodology and effect sizes. Am J Psychiatry 2011 168: 472-85

5 Khandaker GM, Barnett JH, White IR, Jones PB. A quantitative meta-analysis of populationbased studies of premorbid intelligence and schizophrenia. Schizophr Res 2012; 132: 220-7.

6 Deary IJ. Intelligence. Annu Rev Psychol 2012; 63: 453-82.

7 Weschsler D. The Wechsler Adult Intelligence Scale Manual (3rd edn). The Psychological Corporation, 1997.

8 Dickinson D, Goldberg TE, Gold JM, Elvevag B, Weinberger DR. Cognitive factor structure and invariance in people with schizophrenia, their unaffected siblings, and controls. Schizophr Bull 2011; 37: 1157-67.

9 Dickinson D, Ramsey ME, Gold JM. Overlooking the obvious: a meta-analytic comparison of digit symbol coding tasks and other cognitive measures in schizophrenia. Arch Gen Psychiatry 2007; 64: 532-42.

10 Leeson VC, Barnes TR, Harrison M, Matheson E, Harrison I, Mutsatsa SH, et al. The relationship between IQ, memory, executive function, and processing speed in recent-onset psychosis: 1-year stability and clinical outcome. Schizophr Bull 2010; 36: 400-9

11 Penke L, Maniega SM, Bastin ME, Valdes Hernandez MC, Murray C, Royle NA, et al. Brain white matter tract integrity as a neural foundation for general intelligence. Mol Psychiatry 2012; 17: 1026-30.

12 Gray JR, Chabris CF, Braver TS. Neural mechanisms of general fluid intelligence. Nat Neurosci 2003; 6: 316-22.

13 Woolgar A, Parr A, Cusack R, Thompson R, Nimmo-Smith I, Torralva T, et al. Fluid intelligence loss linked to restricted regions of damage within frontal and parietal cortex. Proc Natl Acad Sci U S A 2010; 107: 14899-902.

14 Jung RE, Haier RJ. The Parieto-Frontal Integration Theory (P-FIT) of intelligence: converging neuroimaging evidence. Behav Brain Sci 2007; 30: 135-54.

15 Minzenberg MJ, Laird AR, Thelen S, Carter CS, Glahn DC. Meta-analysis of 41 functional neuroimaging studies of executive function in schizophrenia. Arch Gen Psychiatry 2009; 66: 811-22.

16 Duncan J. The multiple-demand (MD) system of the primate brain: mental programs for intelligent behaviour. Trends Cogn Sci 2010; 14: 172-9. 